\title{
"Drug holiday" in DRONJ (Denosumab Related Osteonecrosis of the Jaws) patients : real benefit in surgical or non surgical therapeutic approach ?
}

\author{
Pierpaolo De Francesco ${ }^{1}$, Marco Frontera ${ }^{1}$, paolo vescovi ${ }^{1}$, Giulia Ghidini ${ }^{1}$, Maddalena Manfredi ${ }^{1}$, Marco Meleti ${ }^{1}$ \\ 1 University of Parma
}

Funding: The author(s) received no specific funding for this work.

Potential competing interests: The author(s) declared that no potential competing interests exist.

\begin{abstract}
One of the most frequent monoclonal antibodies linked to MRONJ is Denosumab. In our study, attention was focused on different therapeutic approaches considering patients treated only with Denosumab or in combination with other drugs associated with the genesis of ONJ. We considered two different clinical approaches: sites treated without surgery; sites treated with traditional surgery or Er:YAG laser $(2940 \mathrm{~nm}, 250 \mathrm{~mJ}, 20 \mathrm{~Hz})$. In our study, the discontinuation of the drug was evaluated in relation to treatment outcomes.
\end{abstract}

\section{Background}

A rare adverse event of pharmacological therapy with anti-resorptive and / or anti-angiogenic drugs is known as medication-related osteonecrosis of the jaw (MRONJ). One of the most frequent monoclonal antibodies linked to MRONJ is Denosumab. In our study, attention was focused on different therapeutic approaches considering patients treated only with Denosumab or in combination with other drugs associated with the genesis of ONJ. We also considered the drug holiday in relation to treatment outcomes ${ }^{[1]}$.

Materials and methods

Thirty-one patients affected by DRONJ are included in the present evaluation. Among these, 22 (71\%) female and 9 (29\%) male, $23(74.2 \%)$ cancer and 8 (25.8\%) non-cancer. Considering the patients included in this study, we analyzed thirty-six DRONJ sites. Among these, 28 (77.8\%) associated to cancer and 8 (22.2\%) non cancer. Based on the AAOMS classification (update 2014), the present study included 1 (2.8\%) site in stage 0, 9 (25\%) sites in stage I, 18 (50\%) in stage II and 8 (22.2\%) in stage III ${ }^{[2]}$. We considered two different clinical approaches: T1: 15 (41.7\%) sites treated without surgery; T2: 21 (58.3\%) treated with traditional surgery or Er:YAG laser (2940nm, 250mJ, 20Hz). T1 and T2 were all treated with Amoxicillin $1 \mathrm{~g}$ twice a day in association with Metronidazole 500mg twice a day for three weeks. We have performed five weekly sessions of low level laser therapy (LLLT, 1064nm, 1.25w, 15 Hz) using Nd:YAG laser. In T1, 11 (73.3\%) sites are localized in patients that have stopped Denosumab two months before our treatment and 4 (26.7\%) in patients that have continued Denosumab. In T2, 14 (66.7\%) sites are localized in patients which have stopped Denosumab two months before treatment and 7 (33.3\%) in patients that have continued Denosumab. 


\section{Results}

In T1, up to $73.3 \%$ of sites improved clinically and up to $33.3 \%$ got complete healing. In T2 $100 \%$ of sites improved clinically and got complete healing. Considering the suspension or not of Denosumab in $\mathrm{T} 1$ : up to $63.6 \%$ of sites that have interrupted Denosumab before treatment improved clinically and up to $36.4 \%$ got complete healing; $100 \%$ of sites that have continued Denosumab improved clinically and up 25\% got complete healing. In T2: $100 \%$ of sites that have interrupted Denosumab improved clinically and got complete healing; similar results were obtained in sites of T2 that have continued Denosumab.

\section{Conclusions}

In the literature there is no unambiguous opinion on how to discontinue the drug before treatment. Considering our study, the surgical treatment is more effective than the conservative approach ${ }^{[3]}$. Discontinuation of Denosumab before treatment does not appear strongly significant for complete healing and especially in clinical improvement (that is a transition from an upper to a lower stage) in the case of a conservative approach. The "drug holiday" does not show significant differences in terms of treatment outcome considering patients treated surgically.

\section{References}

1. 'Camilla Ottesen, Morten Schiodt, Klaus Gotfredsen. (2020). Efficacy of a high-dose antiresorptive drug holiday to reduce the risk of medication-related osteonecrosis of the jaw (MRONJ): A systematic review. Heliyon, vol. 6 (4), e03795. doi:10.1016/j.heliyon.2020.e03795.

2. 'Salvatore L. Ruggiero, Thomas B. Dodson, John Fantasia, Reginald Goodday, et al. (2014). American Association of Oral and Maxillofacial Surgeons Position Paper on Medication-Related Osteonecrosis of the Jaw-2014 Update. Journal of Oral and Maxillofacial Surgery, vol. 72 (10), 1938-1956. doi:10.1016/j.joms.2014.04.031.

3. ^Paolo Vescovi, Maddalena Manfredi, Elisabetta Merigo, Rebecca Guidotti, et al. (2012). Early Surgical Laser-Assisted Management of Bisphosphonate-Related Osteonecrosis of the Jaws (BRONJ): A Retrospective Analysis of 101 Treated Sites with Long-Term Follow-Up. Photomedicine and Laser Surgery, vol. 30 (1), 5-13. doi:10.1089/pho.2010.2955. 